\title{
ON THE FOURIER COEFFICIENTS OF A CONTINUOUS FUNCTION.
}

BY DR. T. H. GRONWALL.

(Read before the American Mathematical Society September 8, 1920.)

It is well known that when

$$
\frac{a_{0}}{2}+\sum_{1}^{\infty}\left(a_{n} \cos n \theta+b_{n} \sin n \theta\right)
$$

is the Fourier expansion of a function $f(\theta)$ which is real and continuous for $0 \leqq \theta \leqq 2 \pi$, then $\Sigma\left(a_{n}{ }^{2}+b_{n}{ }^{2}\right)$ converges. Here the exponent 2 cannot in general be replaced by a smaller one; in fact, Carleman* has constructed an example of a continuous $f(\theta)$ where $\Sigma\left(a_{n}{ }^{2-2 \delta}+b_{n}{ }^{2-2 \delta}\right)$ diverges for any $\delta>0$, and this example has been simplified by Landau. $\dagger$

In the present note it will be shown that, given any singlevalued real function $\varphi(x)$, subject only to the condition that $\varphi(x)$ becomes infinite as $x$ becomes infinite, there exists a real continuous function $f(\theta)$ whose Fourier coefficients $a_{n}, b_{n}$ make the series

$$
\sum\left(a_{n}{ }^{2}+b_{n}^{2}\right) \varphi\left(\frac{1}{a_{n}^{2}+b_{n}^{2}}\right)
$$

divergent. Assuming $\varphi(x)=x^{\delta}$, where $\delta>0$, and observing that $\left(a^{2}+b^{2}\right)^{1-\delta}<a^{2-2 \delta}+b^{2-2 \delta}$, we have the particular result referred to above.

If we denote by $f_{1}(\theta)$ the function conjugate to $f(\theta)$, and write $z=e^{\theta i}, F(z)=f(\theta)+i f_{1}(\theta)$, the Fourier expansion of $F(z)$ is $\Sigma_{0}^{\infty} c_{n} z^{n}$, where $c_{0}=a_{0} / 2, \quad c_{n}=a_{n}-i b_{n} \quad(n>0)$. Our statement will be proved by constructing a function $F(z)$ continuous for $|z|=1$ and such that $\Sigma\left|c_{n}\right|^{2} \varphi\left(1 /\left|c_{n}\right|^{2}\right)$ diverges. This will be done by means of the following result due to Hardy and Littlewood $\ddagger$ and used by Landau, loc. cit., for a different purpose:

* T. Carleman, Ueber die Fourierkoeffizienten einer stetigen Funktion, Acta Math., vol. 41 (1918), pp. 377-384.

$\dagger$ E. Landau, Bemerkungen zu einer Arbeit des Herrn Carleman, MATHEMATISCHE ZeITSCHRIFT, vol. 5 (1919), pp. 147-153.

$\ddagger$ G. H. Hardy and J. E. Littlewood, Some problems of diophantine approximation, Aста Maтн., vol. 37 (1914), pp. 155-239. See p. 220. 
Let $\xi$ be a real irrational number such that all the denominators in its expansion in a continued fraction are bounded (for instance $\xi=\sqrt{2}$ or any quadratic irrationality). Then there exists an $A=A(\xi)$ independent of $n$ and $z$ such that for any $n \geqq 1$, and any $z$ on the unit circle $|z|=1$,

Making

$$
\left|\sum_{\nu=1}^{n} e^{\nu 2 \pi \xi i} z^{\nu}\right|<A \sqrt{n}
$$

$$
F_{\nu}(z)=\sum_{\mu=1}^{n_{\nu}} \frac{e^{\mu^{2} \pi \xi i}}{\sqrt{n_{\nu}}} z^{\mu},
$$

we have therefore $\left|F_{\nu}(z)\right|<A$ for $|z|=1$; writing $k_{\nu}=n_{0}$ $+n_{1}+\cdots+n_{\nu-1}$ and assuming $d_{\nu}$ to be such that $\Sigma\left|d_{\nu}\right|$ converges, we find that the series

$$
F(z)=\sum_{\nu=0}^{\infty} d_{\nu} z^{k_{\nu}} F_{\nu}(z)
$$

converges uniformly for $|z|=1$, so that $F(z)$ is continuous on the unit circle. Multiplying by $z^{-n-1} d z$ and integrating along the unit circle, we may integrate term by term to the right on account of the uniform convergence, and the Fourier coefficients $c_{n}$ of $F(z)$ are thus found to be

$$
c_{n}=d_{\nu} \frac{e^{\mu 2 \pi \xi i}}{\sqrt{n_{\nu}}} \quad\left(n=k_{\nu}+1, k_{\nu}+2, \cdots, k_{\nu}+n_{\nu}\right) .
$$

Consequently

$$
\sum_{n=k_{\nu}+1}^{k_{v+1}}\left|c_{n}\right|^{2} \varphi\left(\frac{1}{\left|c_{n}\right|^{2}}\right)=\left|d_{\nu}\right|^{2} \varphi\left(\frac{n_{\nu}}{\left|d_{\nu}\right|^{2}}\right),
$$

and since $\varphi(x)$ becomes infinite as $x$ becomes infinite, we may choose each $n_{\nu}$ so that

$$
\varphi\left(\frac{n_{\nu}}{\left|d_{\nu}\right|^{2}}\right)>\frac{\left|D_{\nu}\right|}{\left|d_{\nu}\right|^{2}}
$$

where $\Sigma\left|D_{\nu}\right|$ is any given divergent series. With this choice of $n_{\nu}$, it follows that $\Sigma\left|c_{n}\right|^{2} \varphi\left(1 /\left|c_{n}\right|^{2}\right)$ diverges, which proves our theorem.

Technical Staff,

Office of the Chief of Ordnance. 